\title{
Acute and Subacute Effects of Irreversible Electroporation on Normal Common Bile Ducts in a Rabbit Model
}

\section{Qingshan Li}

the first affiliated hospital of xi'an jiaotong university

\section{Fenggang Ren}

The first affiliated hospital of xi'an jiaotong university

\section{YuChi Zhang}

The first affiliated hospital of xi'an jiaotong university

\section{Pengkang Chang}

The first affiliated hospital of xi'an jiaotong university

\section{Yue Wang}

The first affiliated hospital of xi'an jiaotong university

\section{$\mathrm{Tao} \mathrm{Ma}$}

The first affiliated hospital of xi'an jiaotong university

\section{Liangshuo Hu}

The first affiliated hospital of xi'an jiaotong university

\section{Rongqian Wu}

The first affiliated hospital of xi'an jiaotong university

\section{Bo Wang}

The first affiliated hospital of xi'an jiaotong university

Yi Lv (D luyi169@126.com )

https://orcid.org/0000-0003-3636-6664

\section{Research article}

Keywords: acute and subacute effect, irreversible electroporation, bile duct

Posted Date: March 16th, 2020

DOI: https://doi.org/10.21203/rs.3.rs-17340/v1

License: (c) (1) This work is licensed under a Creative Commons Attribution 4.0 International License. Read Full License 
Version of Record: A version of this preprint was published at Journal of Hepato-Biliary-Pancreatic Sciences on August 15th, 2020. See the published version at https://doi.org/10.1002/jhbp.807. 


\section{Abstract}

Background This study aimed to evaluate the acute and subacute effects of irreversible electroporation (IRE) on normal common bile ducts (CBDs) in a rabbit model. Methods Cell susceptibility to IRE was assessed in vitro with cholangiocarcinoma and normal cell lines at varying voltages. The distributions of electric field and temperature were evaluated with a two-dimensional simulation model of common bile duct. In vivo bile duct IRE ablation was performed in 28 adult New Zealand rabbits. Liver function assessments and cholangiography were performed post-IRE to evaluate biliary complications. The biliary tract was extracted en bloc and histopathological analyses were performed using hematoxylin-eosin (H\&E) and Masson trichrome (MT) staining, and terminal deoxynucleotidyl transferase-mediated nick-end labeling (TUNEL) assay. Results Different cells showed different susceptibility to the effect of IRE, cancer cell line HUCC-T1 was the least sensitive to IRE. Simulations predicted the distributions of electric field and temperature during IRE process, and the maximum temperature of tissue was below $43^{\circ} \mathrm{C}$. In all animals, biliary dilatation and stricture were observed in $4 / 28$ animals respectively by postoperative days 7,14 , and 28 . H\&E staining revealed complete cell death with inflammatory infiltration, edema, and hemorrhage. Bile duct epithelial recovery was completed between post-IRE days 7-28. Conclusions The normal CBD retains the lumen wall integrity and function following IRE with immediate periductal placement of the electrode. However, the parameters of cholangiocarcinoma IRE ablation need to be determined more precisely to ensure the treatment efficacy and reduce the risk of collateral damage.

\section{Background}

Malignant biliary tumors are uncommon and usually diagnosed following malignant biliary obstruction $[1-3]$. Surgical resection is currently the only potential curative treatment for early recognition and diagnosis. However, a majority of patients present late in the course of disease with only $20-40 \%$ resectable cases due to the advanced tumor stage at the time of diagnosis, and of these potentially resectable tumors, $R_{0}$ resection is not always achieved [4-6]. Therefore, placement of biliary stents is typically chosen as palliative management for biliary decompression with an estimated life expectancy of approximately 3-6 months [7]. Compared with surgical interventions, biliary stents offer shorter durations of hospitalization, lower overall costs, and lower morbidity; however, maintenance of stent patency is difficult because of tumor ingrowth or overgrowth $[8,9]$.

Tissue ablation techniques such as radiofrequency ablation (RFA) can safely induce tumor necrosis and are successfully used in early stage cancers or patients unfit for surgery. Recently, endoscopic or percutaneous focal ablation has been piloted to prolong ductal patency and debulk ingrowing tumors [10]. Intraductal RFA with an endobiliary catheter (Habib EndoHPB; EMcision, London, UK) has been designed to potentially improve the stent patency and survival rates $[11,12]$. However, because of heat sink and thermal injury, its clinical use was confined by the risk of treatment-related complications and limited efficacy[13-15]. 
Irreversible electroporation (IRE) is a non-thermal ablation technique that was first introduced for tumor ablation in $2005[16,17]$. In contrast to other thermal ablation techniques, IRE can achieve non-thermal ablation through a high-voltage pulsed electric field without destruction of the extracellular matrix (ECM) $[18,19]$. Therefore, IRE holds promise as an endoscopic ablation option in patients with malignant biliary obstruction. Various retrospective studies have demonstrated the safety of peri-biliary tumor ablation with IRE [20, 21], and catheter-directed IRE in swine bile duct was designed for biliary malignancies [22]. However, the effects of IRE on the bile duct deserve further investigation to determine the potential thermal injury and the long-term safety and efficiency. Thus, the purpose of this study was to evaluate the incidence of treatment related adverse events after common bile duct IRE ablation and the temperature distribution during the treatment period.

\section{Methods}

\section{In vitro IRE experimental protocols}

Three human cholangiocarcinoma cell lines (HUCC-T1, HCCC9810, and QBC939) and the human bile duct epithelium cell line (HIBEpiC) were used to assess the effects of in vitro IRE. All cells were treated with 70 pulses of high pulsed electric field with 90 microseconds duration at varying voltages. CCK-8 assay (Dojindo, Japan) were performed to assess the cell viability changes 24 hours after IRE treatment. Detailed cell culture methods and IRE parameter were described in appendix 1 in supplemental materials.

\section{Computer simulation of in vivo IRE}

A two-dimensional model of common bile duct wall containing the epithelium layer (EL) and subepithelium connective tissue layer (SL) were constructed in this study (Figure 1A). Computer simulations were performed to estimate the distribution of electric field and identify the potential of thermal injury during IRE treatment with a plate electrode. Details of the model construction were described in appendix 2 (supplementary materials) [23].

\section{In vivo evaluation of bile duct IRE}

The animal experiments were performed according to the protocol approved by the Institutional Animal Care and Use Committee of Xi'an jiaotong University in accordance with ARRIVE guidelines and the National Institutes of Health guide for the care and use of laboratory animals. 28 adult male New Zealand rabbits (weight: $2.0-3.5 \mathrm{~kg}$ ) provided by Experimental Animal Center of Xi'an Jiaotong University were included for bile duct IRE ablation.

All animals were maintained in a fasting state for 24 hours before preoperatively. After anesthesia, animals were placed in the supine position on the operation table and IRE ablation was conducted through an open method. Animals were euthanized at 1 day $(n=4), 3$ days $(n=4), 7$ days $(n=7), 14$ days $(n=7)$, and 28 days $(n=6)$ after IRE procedure respectively by pentobarbital sodium excessive anesthesia $(90 \mathrm{mg} / \mathrm{kg}$ ) for sample acquisition. Cholangiography was performed before euthanize to evaluate the 
incidence of adverse events post-IRE. In vivo experiment details were described in appendix 3 (supplementary materials).

\section{Gross and Histopathological analysis}

The animals were euthanized on either post-IRE day $1,3,7,14$, or 28 via excessive anesthesia. The common bile duct was removed en bloc and dissected longitudinally to expose the mucosal surface. The lesions along with the adjacent normal tissue were fixed in formalin, and the slides were stained with hematoxylin and eosin (H\&E) and Masson trichrome (MT) stains. Terminal-deoxynucleoitidyl transferase mediated nick end labeling (TUNEL) assay was performed according to the manufacturer's instructions to identify cell apoptosis. The slides were scanned with a digital slide scanner (Pannoramic SCAN II; 3DHISTECH Ltd., Budapest, Hungary) and analyzed with CaseViewer 2.0 (3DHISTECH Ltd.). Histopathologic analyses were performed by a pathologist with 5 years of experience.

\section{Statistical Analysis}

Data were reported as mean \pm standard error (SE). Statistical differences were analyzed with the parametric or non-parametric unpaired Student's t-test. SPSS version 22.0 for Windows (IBM Inc., Armonk, NY, USA) was used for the statistical analyses and $p$-value $<0.05$ was considered statistically significant.

\section{Results}

\section{Sensitivity of different cell lines to the effect of IRE}

Three cholangiocarcinoma cell lines and one normal bile duct epithelium cell line were treated with IRE at different electric field strength. The cell viability decreased in a dose dependent fashion according to the applied electric field strength (Table 1). However, different cells showed different susceptibility to the effect of IRE. Compared to HCCC-9810 and QBC-939, the normal bile duct epithelium cell line HIBEpiC seemed more resistant to IRE treatment, and HUCC-T1 cells were least sensitive to IRE. The IC50 value was 1085.0 V/cm, 951.0 V/cm, 953.8 V/cm, 1372.0 V/cm for HIBEpiC, QBC-939, HCCC-9810, and HUCCT1 respectively (Figure 2). Therefore, the electric field strength $1500 \mathrm{~V} / \mathrm{cm}$ was chosen for further computer simulation and in vivo evaluation.

\section{Distributions of electric field and temperature}

The distributions of electric field and temperature during bile duct IRE ablation were shown in Figure 1. The electric field and temperature were distributed unevenly in the EL and SL due to the difference of electrical and thermal properties. The electric field strengthen was higher in the epithelium layer and the corner of the electrode-tissue interface (Figure 1B). IRE ablation caused an increase in tissue temperature, and the maximum temperature after IRE procedure was observed at the corner of the electrode-tissue 
interface (Figure 1C-D). However, the maximum temperature of the entire area remained below $43^{\circ} \mathrm{C}$ which was considered the threshold of thermal injury (Figure 1E) during IRE ablation period.

\section{In vivo IRE ablation of normal common bile duct}

IRE of the common bile duct was successfully performed in 28 animals without any interruption and without bleeding, or extravasation and dilatation immediately post-IRE. Pulse delivery was completed within 2 minutes in each treatment process, and all the animals survived the IRE procedure.

During the follow-up period, 2 animals died (1 each in the 7-and 14-day groups) due to severe abdominal infections on post-IRE ablation day 6 . During autopsy, no IRE-procedure related biliary adverse events were observed. Biliary dilatation without apparent stenosis was first identified on post-operative day 7 and in a total of 4 animals throughout the follow-up duration. Furthermore, 4 animals experienced biliary stricture, which was also first observed on post-operative day 7 (Table 2). Cholangiography confirmed these biliary complications and revealed leakage free passage of contrast in the treated bile ducts of these animals (Figure 3). No perforation of the bile duct was observed. Laboratory values showed an early increase in serum alanine and aspartate transaminases (ALT and AST) on the first post-IRE day, and they rapidly returned to normal levels within one week. Similarly, gamma-glutamyl transferase ( $\mathrm{y}-\mathrm{GT})$ and alkaline phosphatase (ALP) levels were increased in the first week post-IRE and gradually returned to normal levels over the duration of the follow-up period. There was no significant increase in serum bilirubin levels after the ablation (Supplementary Figure 2).

\section{Histopathology}

Histopathologic analysis revealed epithelial sloughing and complete transmural cell ablation of normal common bile duct wall following IRE (Figure 4A). H\&E staining revealed sharp and clear boundary between the ablated area and the surrounding normal tissue day 1 post-IRE. Full-thickness cell necrosis with hyperemia and infiltration of inflammatory cells was observed in the treatment region (Figure 4A-B). The extracellular matrix (ECM) within the treatment region demonstrated inflammation, and MT staining indicated intactness of ECM in the ablated area (Figure 4C). In TUNEL assay, the presence of diffuse positive cells in the ablated area demonstrated apoptotic cell death in the common bile duct wall at day 1 post-IRE (Figure 4D). Tissue samples in the control group had histologic appearances of normal bile duct wall. Samples on day 3 demonstrated similar appearance (Figure 5A-B). At day 7 post-IRE, diffuse fibrosis was observed in the treatment region with the presence of mucosal ulceration. Regeneration of the bile duct epithelium was completed between days 14 and 28 post-IRE (Figure $5 \mathrm{C}-\mathrm{H}$ ).

\section{Discussion}

IRE has emerged as a promising non-thermal tissue ablation technique in the last few decades. Due to the advantages of achieving tumor ablation without heat sink and ECM destruction, IRE is drawing more and more attention for its potential in the treatment of tumors of hollow viscera or close to major anatomical structures [23-26]. In the present study, the effect of IRE on normal rabbit common bile duct 
was evaluated directly. In vitro cell experiments were conducted to determine the appropriate electric field parameter for simulation and in vivo bile duct IRE ablation. We found that bile duct IRE ablation was feasible without dramatic temperature increase using an appropriate parameter, however the risk of biliary dilatation and stricture may reduce the biliary patency in the long-term run.

Results from in vitro experiments of this study confirmed the sensitivity difference of different cell lines to the effect of IRE. This observation was consistent with previous studies. Gianulis et al. found that the difference in $L D_{50}$ spanned as much as nearly 80-fold among different cell types. Besides, they also found that the different sensitivity showed no correlation with cell or nuclear size, cell morphology, and metabolism level [27]. Kodama et al. observed that cancer cell lines seemed more sensitive to cell death from IRE compared to normal human bronchial epithelial cell line [28]. Our results showed that the normal cell line HIBEpiC seemed more resistant to IRE treatment than cancer cell line HCCC-9810 and QBC-939. However, HUCC-T1 cancer cell were the least sensitive to IRE. Thus, the different sensitivity may not be determined simply by benign or malignant characteristics. The mechanism of IRE susceptibility needs to be further investigated to provide evidence for optimal parameter selection.

Previous studies have evaluated the effects of IRE on vital structures adjacent to the target ablation area [29-31]. Kevin et al. found that IRE was safe and effective for liver ablation in the liver hilum without the presence of heat sink, and that major bile ducts and portal veins were more resistant to IRE ablation [30]. Furthermore, long-term increase in serum bilirubin or transaminases levels was not identified, which was similar to our findings. Choi et al. evaluated the chronic effects of IRE on bile ducts and found that $6 / 7$ animals in the periductal (electrode-to-duct distance $\leq 2 \mathrm{~mm}$ ) electrode placement group developed bile duct narrowing, but no bile duct narrowing was observed when the electrode was placed more than $2 \mathrm{~mm}$ from the bile duct [31]. However, the parameters used in these studies were based on the clinical experience, and the electric field distribution and temperature changes during IRE procedure were not evaluated. Our study demonstrated that the electric field strength should be above $1500 \mathrm{~V} / \mathrm{cm}$ to achieve cholangiocarcinoma cell ablation. The simulation models showed the uneven distributions of electric field and temperature in different bile duct layer because of the difference in electric and thermal properties [32], this may illustrate the resistance discrepancy of different tissues. In addition, computer simulation revealed that the temperature was not dramatic increased during the ablation process, which may not cause thermal injury.

As for catheter-directed biliary ablation, Ueshima et al. performed normal porcine common bile duct ablation with IRE with endoscopy. Their results illustrated the feasibility and early safety within one week after catheter-directed IRE [22]. However, the chronic effects were not evaluated in their study.

Srimathveeravalli et al. assessed the effects of IRE on normal porcine ureters through catheter-mounted electrode. They found that the intraluminal catheter-directed IRE ablation was safe and effective in normal ureter ablation over a relatively short follow-up period. However, the ureteral strictures were observed on day 7 post-IRE and did not resolve by day 28 [33,34]. Even though the effects of IRE may vary due to the variations in the geometry of the electrode and the parameters of IRE, the presence of biliary stricture and dilatation in the present study and in that by Choi et al. suggests that bile duct injury 
always manifested as a delayed effect and the long-term effects of catheter-directed IRE on the bile ducts need further investigations.

There are several limitations to this study. The parameters of the in vitro experiments only changed the voltage, the pulse duration and number should also be assessed to obtain optimal treatment parameter in future studies. Besides, the computer simulation models can partly reveal the temperature distribution, however the change of tissue temperature was not measured in real time. The thermal injury may also exist because of the complex environment of in vivo study as reported in previous studies [34]. Furthermore, we performed our study on normal rabbit common bile duct rather than a tumor model, electrical properties and microenvironments are different between the tumor and normal tissue; tumor ablation with IRE may require different parameters and have different effects. Therefore, further studies are needed to optimize the treatment parameters tumor ablation.

In conclusion, the normal common bile duct retains the lumen wall integrity and function following IRE with immediate periductal placement of the electrode. However, the parameters of cholangiocarcinoma IRE ablation need to be determined more precisely to ensure the treatment efficacy and reduce the risk of collateral damage.

\section{Abbreviations}

Irreversible electroporation

IRE

Normal common bile ducts

CBDs

Radiofrequency ablation

RFA

Extracellular matrix

ECM

Epithelium layer

EL

Sub-epithelium connective tissue layer

SL

Alanine transaminases

ALT

Aspartate transaminases

AST

Gamma-glutamyl transferase

Y-GT

Alkaline phosphatase

ALP

Hematoxylin and eosin 
HE

Masson trichrome

MT

Terminal-deoxynucleoitidyl transferase mediated nick end labeling

TUNEL

\section{Declarations}

\section{Ethics approval}

The animal experiments were performed according to the protocol approved by the Institutional Animal Care and Use Committee of Xi'an jiaotong University.

\section{Consent for publication}

Not applicable

\section{Availability of data and materials}

All data generated or analysed during this study are included in this published article and its supplementary information files.

\section{Competing interests}

The authors declare that they have no competing interests.

\section{Funding}

This work was supported by: 1. National Natural Science Foundation of China (Grant No.81727802) (design of the study and interpretation of data); 2. National Key R\&D Project of China (Grant No. 2018YFC0115300) (in vivo experiments data collection and analysis); 3 . The Fundamental Research Funds for the Central Universities (Grant No. XJH012019076) (in vitro data analysis)

\section{Authors' contributions}

B.W. and Y.L. contributed to study concept and design; QS.L., FG.R., Y.W., T.M. and P.CH. contributed to animal surgery and data statistics; YC. ZH. and LS. H. contributed to invitro experiments; RQ. W. contributed to the study supervision.

\section{Acknowledgements}

We kindly express our appreciation to staffs of Experimental Animal Center of Xi'an Jiaotong University for their assistance of animal care. 


\section{References}

1. Skipworth JR, Olde Damink SW, Imber C, Bridgewater J, Pereira SP, Malago M: Review article: surgical, neo-adjuvant and adjuvant management strategies in biliary tract cancer. Aliment Pharmacol Ther 2011, 34(9):1063-1078.

2. Mensah ET, Martin J, Topazian M: Radiofrequency ablation for biliary malignancies. Curr Opin Gastroenterol 2016, 32(3):238-243.

3. Cui W, Fan W, Lu M, Zhang Y, Yao W, Li J, Wang Y: The safety and efficacy of percutaneous intraductal radiofrequency ablation in unresectable malignant biliary obstruction: A single-institution experience. BMC cancer 2017, 17(1).

4. Strand DS, Cosgrove ND, Patrie JT, Cox DG, Bauer TW, Adams RB, Mann JA, Sauer BG, Shami VM, Wang AY: ERCP-directed radiofrequency ablation and photodynamic therapy are associated with comparable survival in the treatment of unresectable cholangiocarcinoma. Gastrointest Endosc 2014, 80(5):794-804.

5. Alvarez-Sanchez MV, Napoleon B: Review of endoscopic radiofrequency in biliopancreatic tumours with emphasis on clinical benefits, controversies and safety. World J Gastroentero/ 2016, 22(37):8257-8270.

6. Brandi G, Venturi M, Pantaleo MA, Ercolani G, Gico: Cholangiocarcinoma: Current opinion on clinical practice diagnostic and therapeutic algorithms: A review of the literature and a long-standing experience of a referral center. Digestive and liver disease : official journal of the Italian Society of Gastroenterology and the Italian Association for the Study of the Liver 2016, 48(3):231-241.

7. Perdue DG, Freeman ML, DiSario JA, Nelson DB, Fennerty MB, Lee JG, Overby CS, Ryan ME, Bochna GS, Snady HW et al: Plastic versus self-expanding metallic stents for malignant hilar biliary obstruction: a prospective multicenter observational cohort study. Journal of clinical gastroenterology 2008, 42(9):1040-1046.

8. Loew BJ, Howell DA, Sanders MK, Desilets DJ, Kortan PP, May GR, Shah RJ, Chen YK, Parsons WG, Hawes RH et al: Comparative performance of uncoated, self-expanding metal biliary stents of different designs in 2 diameters: final results of an international multicenter, randomized, controlled trial. Gastrointest Endosc 2009, 70(3):445-453.

9. Ridtitid W, Rerknimitr R: Management of an occluded biliary metallic stent. World journal of gastrointestinal endoscopy 2012, 4(5):157-161.

10. Wallace MB, Wang KK, Adler DG, Rastogi A: Recent Advances in Endoscopy. Gastroenterology 2017, 153(2):364-381.

11. Wang AY, Yachimski PS: Endoscopic Management of Pancreatobiliary Neoplasms. Gastroenterology 2018, 154(7):1947-1963.

12. Rustagi T, Jamidar PA: Intraductal radiofrequency ablation for management of malignant biliary obstruction. Dig Dis Sci 2014, 59(11):2635-2641. 
13. Sofi AA, Khan MA, Das A, Sachdev M, Khuder S, Nawras A, Lee W: Radiofrequency ablation combined with biliary stent placement versus stent placement alone for malignant biliary strictures: a systematic review and meta-analysis. Gastrointest Endosc 2018, 87(4):944-951.e941.

14. Cho JH, Jeong S, Kim EJ, Kim JM, Kim YS, Lee DH: Long-term results of temperature-controlled endobiliary radiofrequency ablation in a normal swine model. Gastrointestinal Endoscopy 2018, 87(4):1147-1150.

15. Zhou C, Wei B, Gao KUN, Zhai R: Biliary tract perforation following percutaneous endobiliary radiofrequency ablation: A report of two cases. Oncology Letters 2016, 11(6):3813-3816.

16. Davalos RV, Mir IL, Rubinsky B: Tissue ablation with irreversible electroporation. Annals of biomedical engineering 2005, 33(2):223-231.

17. Miller L, Leor J, Rubinsky B: Cancer cells ablation with irreversible electroporation. Technology in cancer research \& treatment 2005, 4(6):699-705.

18. Phillips M, Maor E, Rubinsky B: Nonthermal irreversible electroporation for tissue decellularization. Journal of biomechanical engineering 2010, 132(9):091003.

19. Ren F, Li Q, Hu L, Yan X, Gao Z, Zhang J, Gao W, Zhang Z, Chang P, Chen X et al: Safety and efficacy of magnetic anchoring electrode-assisted irreversible electroporation for gastric tissue ablation. Surgical endoscopy 2019.

20. Narayanan G, Bhatia S, Echenique A, Suthar R, Barbery K, Yrizarry J: Vessel patency post irreversible electroporation. Cardiovasc Intervent Radio/ 2014, 37(6):1523-1529.

21. Cannon R, Ellis S, Hayes D, Narayanan G, Martin RC, 2nd: Safety and early efficacy of irreversible electroporation for hepatic tumors in proximity to vital structures. Journal of surgical oncology 2013, 107(5):544-549.

22. Ueshima E, Schattner M, Mendelsohn R, Gerdes H, Monette S, Takaki H, Durack JC, Solomon SB, Srimathveeravalli G: Transmural ablation of the normal porcine common bile duct with catheterdirected irreversible electroporation is feasible and does not affect duct patency. Gastrointestinal Endoscopy 2018, 87(1):300.e301-300.e306.

23. Ren F, Li Q, Gao X, Zhu K, Zhang J, Chen X, Yan X, Chu D, Hu L, Gao Z et al: Electrical and thermal analyses of catheter-based irreversible electroporation of digestive tract. Int J Hyperthermia 2019, 36(1):854-867.

24. Silk MT, Wimmer T, Lee KS, Srimathveeravalli G, Brown KT, Kingham PT, Fong Y, Durack JC, Sofocleous CT, Solomon SB: Percutaneous ablation of peribiliary tumors with irreversible electroporation. J Vasc Interv Radio/ 2014, 25(1):112-118.

25. Srimathveeravalli G, Wimmer T, Monette S, Gutta NB, Ezell PC, Maybody M, Weiser MR, Solomon SB: Evaluation of an endorectal electrode for performing focused irreversible electroporation ablations in the Swine rectum. J Vasc Interv Radiol 2013, 24(8):1249-1256.

26. Li J, Zeng J, Chen J, Shi J, Luo X, Fang G, Chai W, Zhang W, Liu T, Niu L: Evaluation of the safety of irreversible electroporation on the stomach wall using a pig model. Exp Ther Med 2017, 14(1):696702. 
27. Gianulis EC, Labib C, Saulis G, Novickij V, Pakhomova ON, Pakhomov AG: Selective susceptibility to nanosecond pulsed electric field (nSPEF) across different human cell types. Cellular and Molecular Life Sciences 2017, 74(9):1741-1754.

28. Kodama H, Vroomen LG, Ueshima E, Reilly J, Brandt W, Paluch LR, Monette S, Jones D, Solomon SB, Srimathveeravalli G: Catheter-based endobronchial electroporation is feasible for the focal treatment of peribronchial tumors. The Journal of thoracic and cardiovascular surgery 2018, 155(5):21502159.e2153.

29. Dollinger M, Zeman F, Niessen C, Lang SA, Beyer LP, Muller M, Stroszczynski C, Wiggermann P: Bile Duct Injury after Irreversible Electroporation of Hepatic Malignancies: Evaluation of MR Imaging Findings and Laboratory Values. J Vasc Interv Radio/ 2016, 27(1):96-103.

30. Charpentier KP, Wolf F, Noble L, Winn B, Resnick M, Dupuy DE: Irreversible electroporation of the liver and liver hilum in swine. HPB : the official journal of the International Hepato Pancreato Biliary Association 2011, 13(3):168-173.

31. Choi JW, Lu DS, Osuagwu F, Raman S, Lassman C: Assessment of chronological effects of irreversible electroporation on hilar bile ducts in a porcine model. Cardiovasc Intervent Radiol 2014, 37(1):224-230.

32. Hasgall PA DGF, Baumgartner C, Neufeld E, Lloyd B, Gosselin MC, Payne D, Klingenböck A, Kuster N: IT'IS Database for thermal and electromagnetic parameters of biological tissues," Version 4.0. May 15, 2018.

33. Srimathveeravalli G, Silk M, Wimmer T, Monette S, Kimm S, Maybody M, Solomon SB, Coleman J, Durack JC: Feasibility of catheter-directed intraluminal irreversible electroporation of porcine ureter and acute outcomes in response to increasing energy delivery. J Vasc Interv Radio/ 2015, 26(7):10591066.

34. Srimathveeravalli G, Cornelis F, Wimmer T, Monette S, Kimm SY, Maybody M, Solomon SB, Coleman JA, Durack JC: Normal Porcine Ureter Retains Lumen Wall Integrity but Not Patency Following Catheter-Directed Irreversible Electroporation: Imaging and Histologic Assessment over 28 Days. J Vasc Interv Radiol 2017, 28(6):913-919 e911.

\section{Tables}


Table 1. Cell viability following irreversible electroporation at different range of electric field.

\begin{tabular}{ccccc}
\hline \multirow{2}{*}{ Electric Field (V/cm) } & \multicolumn{4}{c}{ Cell viability $($ Mean \pm SD, \%) } \\
& HUCC-T1 & HCCC-9810 & QBC-939 & HIBEpiC \\
\hline 500 & $97.38 \pm 2.37$ & $92.62 \pm 3.78$ & $95.71 \pm 3.30$ & $92.51 \pm 2.76$ \\
750 & $87.53 \pm 4.05$ & $89.58 \pm 3.57$ & $93.51 \pm 5.17$ & $82.62 \pm 3.79$ \\
1000 & $86.71 \pm 2.80$ & $38.52 \pm 5.66$ & $40.15 \pm 5.49$ & $67.66 \pm 3.34$ \\
1250 & $76.43 \pm 5.68$ & $12.48 \pm 1.83$ & $14.77 \pm 2.69$ & $20.53 \pm 1.04$ \\
1500 & $26.53 \pm 3.40$ & $10.96 \pm 0.58$ & $13.91 \pm 1.14$ & $14.57 \pm 4.91$ \\
1750 & $16.16 \pm 3.24$ & $4.82 \pm 1.30$ & $6.67 \pm 0.89$ & $8.79 \pm 3.63$ \\
2000 & $9.26 \pm 2.85$ & $2.58 \pm 1.00$ & $4.91 \pm 1.33$ & $8.40 \pm 3.93$
\end{tabular}

Table 2. Postoperative status of bile duct of different groups

\begin{tabular}{lccccc}
\hline Postoperative status & $\begin{array}{c}\text { POD1 } \\
(\mathrm{n}=4)\end{array}$ & $\begin{array}{c}\text { POD3 } \\
(\mathrm{n}=4)\end{array}$ & $\begin{array}{c}\text { POD7 } \\
(\mathrm{n}=7)\end{array}$ & $\begin{array}{c}\text { POD14 } \\
(\mathrm{n}=7)\end{array}$ & $\begin{array}{c}\text { POD28 } \\
(\mathrm{n}=6)\end{array}$ \\
\hline Normal & 4 & 4 & 4 & 3 & 3 \\
Biliary dilatation & 0 & 0 & 1 & 2 & 1 \\
Biliary stricture & 0 & 0 & 1 & 1 & 2 \\
Biliary perforation & 0 & 0 & 0 & 0 & 0 \\
\hline Postoperative death & 0 & 0 & 1 & 1 & 0 \\
\hline
\end{tabular}

\section{Supplemental Figures}

Supplementary Figure 1. The tweezer electrodes and intra-operative image. A. The electrode (Platinum tweezertrode, 7mm diameter, BTX, USA). B. The exposure of porta hepatis. C. Intra-operative image of IRE procedure.

Supplementary Figure 2. The variation of Laboratory values duration the follow-up period. A. ALT. B. AST. C. DBIL. D. TBIL. E. Y-GT. F. ALP.

\section{Figures}


A

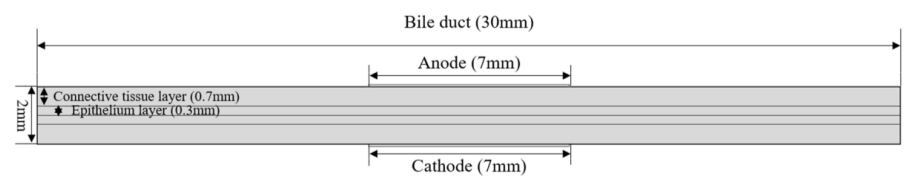

$\mathrm{C}$

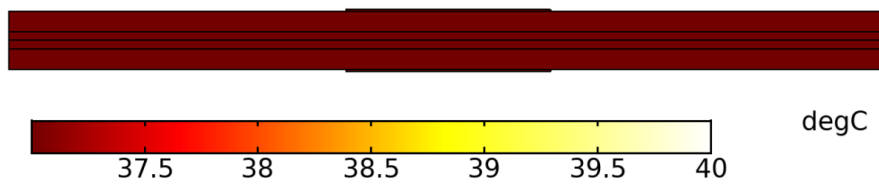

B

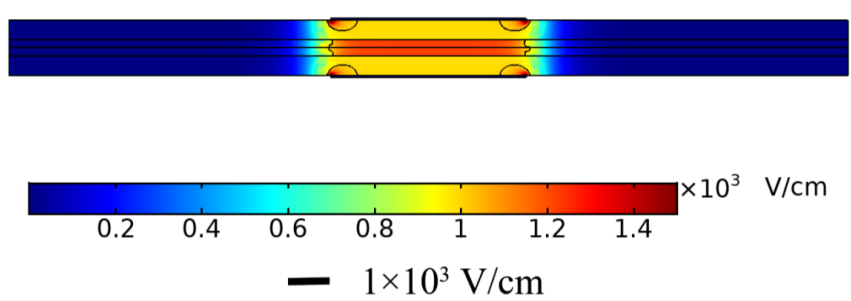

$\mathrm{D}$

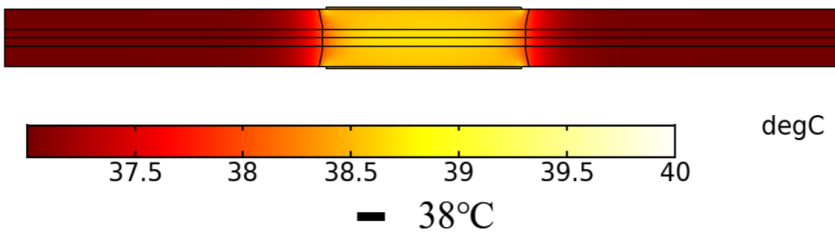

E

\section{Maximum Temperature}

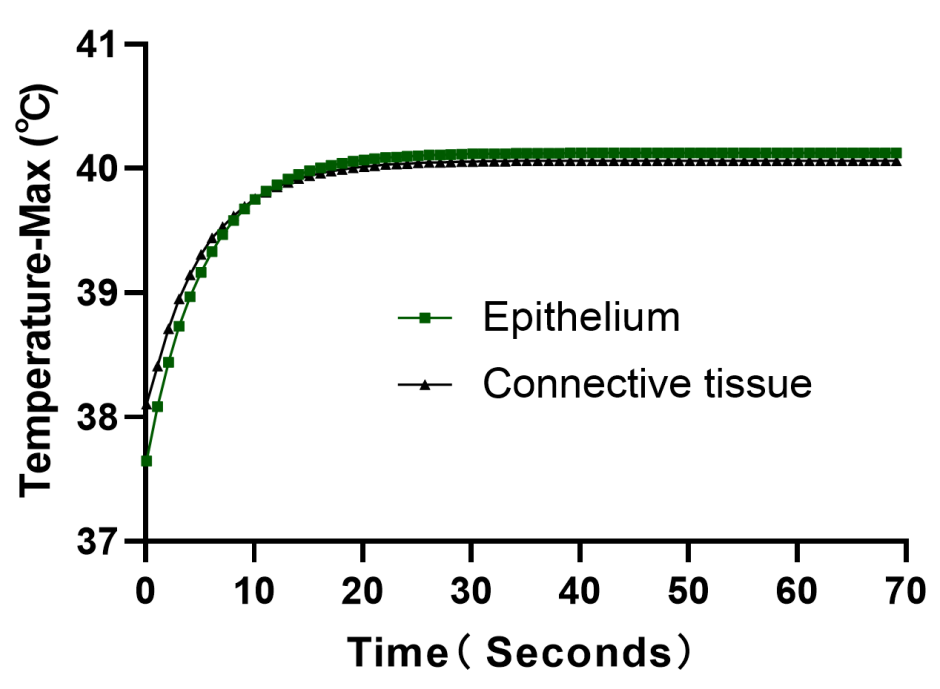

\section{Figure 1}

The computer simulation model of bile duct ablation. A. two-dimensional model of bile duct wall. B. Electric field distribution during pulse delivery with plate electrode. C. Tissue temperature at the beginning of the first pulse. D. Tissue temperature at the end of pulse delivery. E. The change of the maximum temperature during pulse delivery. 
HIBEpiC

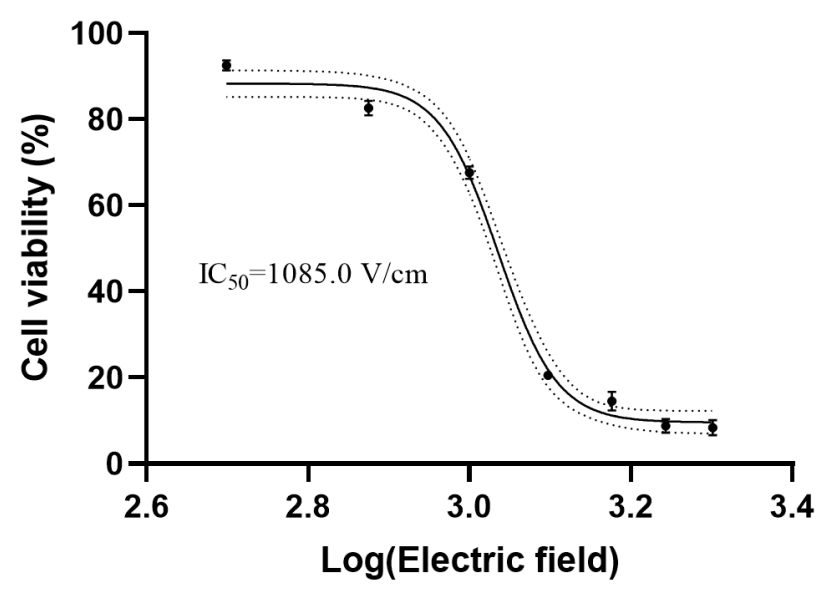

HCCC-9810

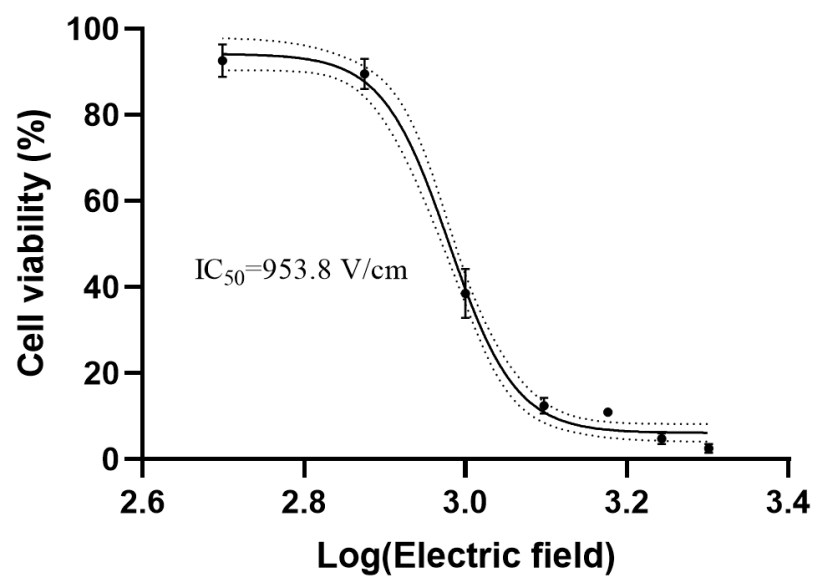

QBC939

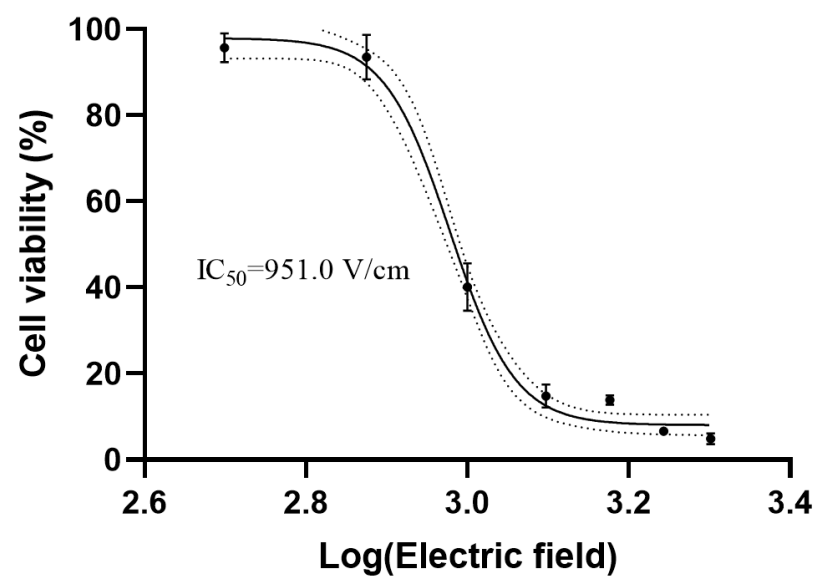

HuCC-T1

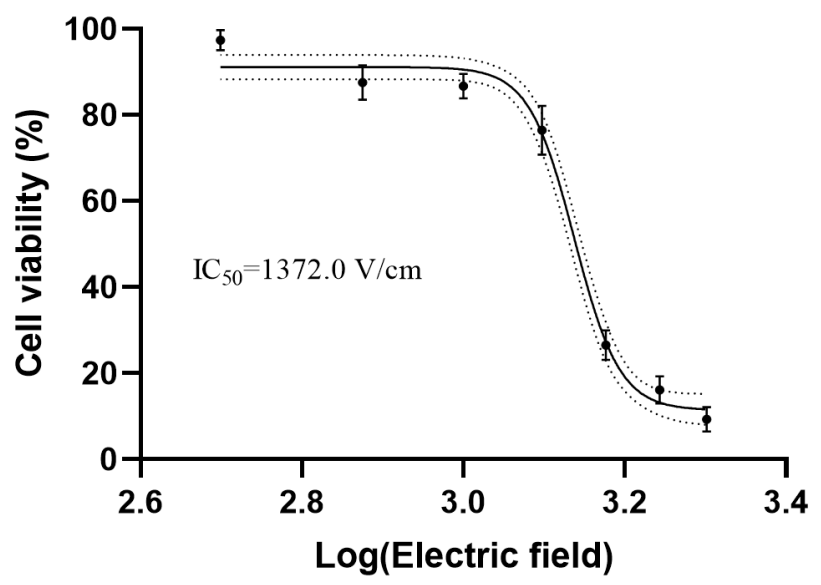

Cell viability following in vitro irreversible electroporation

Figure 2

Cell viability following in vitro irreversible electroporation of different cell lines. A. Normal human bile duct epithelium cell line HIBEpiC. B. Cancer cell line QBC-939. C. Cancer cell line HCCC-9810. D. Cancer cell line HUCC-T1. 

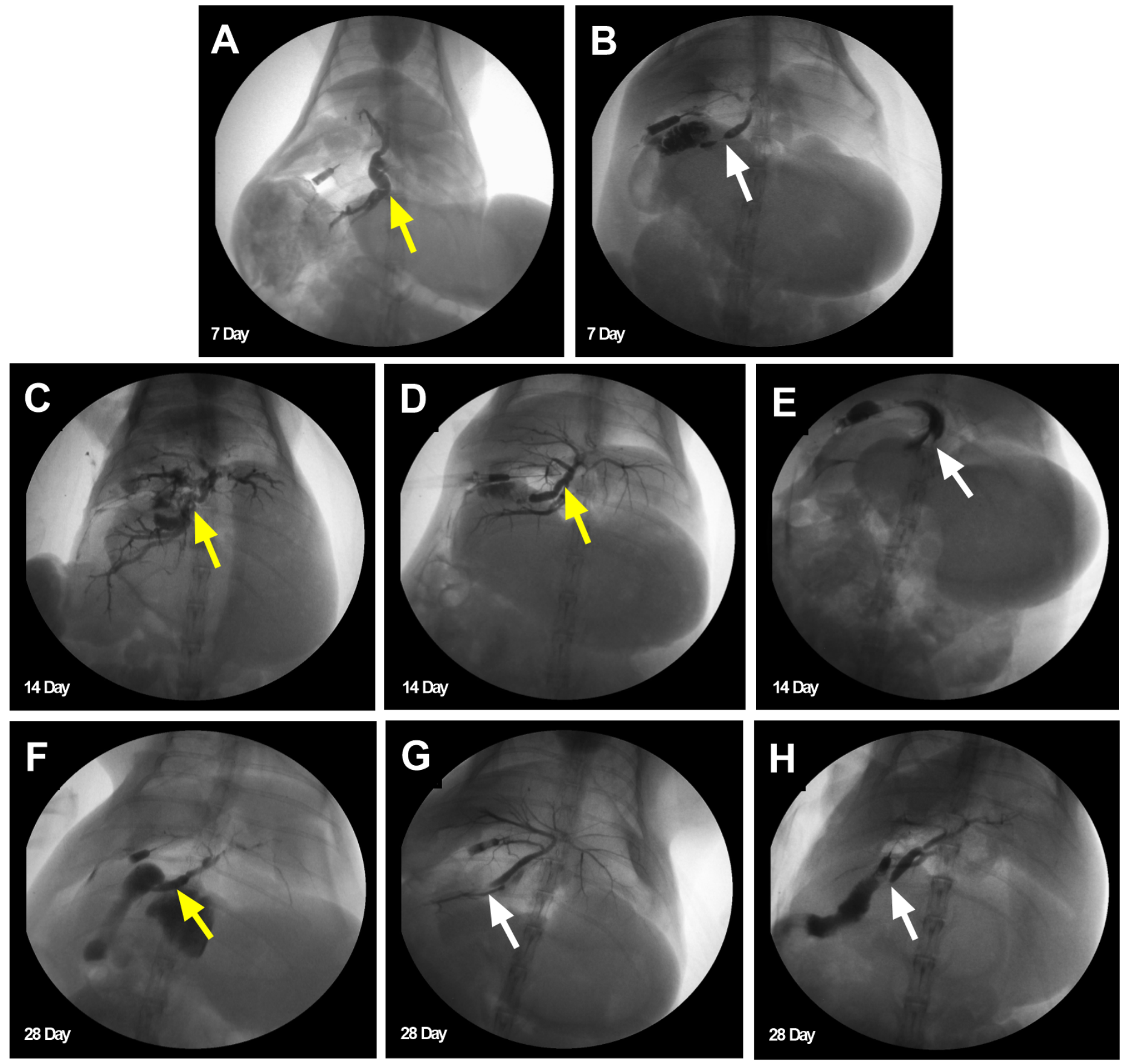

Figure 3

Cholangiography of animals with biliary complication. The biliary dilatation (Yellow arrow) occurred on day $7(A), 14(C, D), 28$ (F) post-IRE. The biliary stricture (White arrow) observed on day 7 (B), 14 (E), 28 (G, H) post-IRE. 


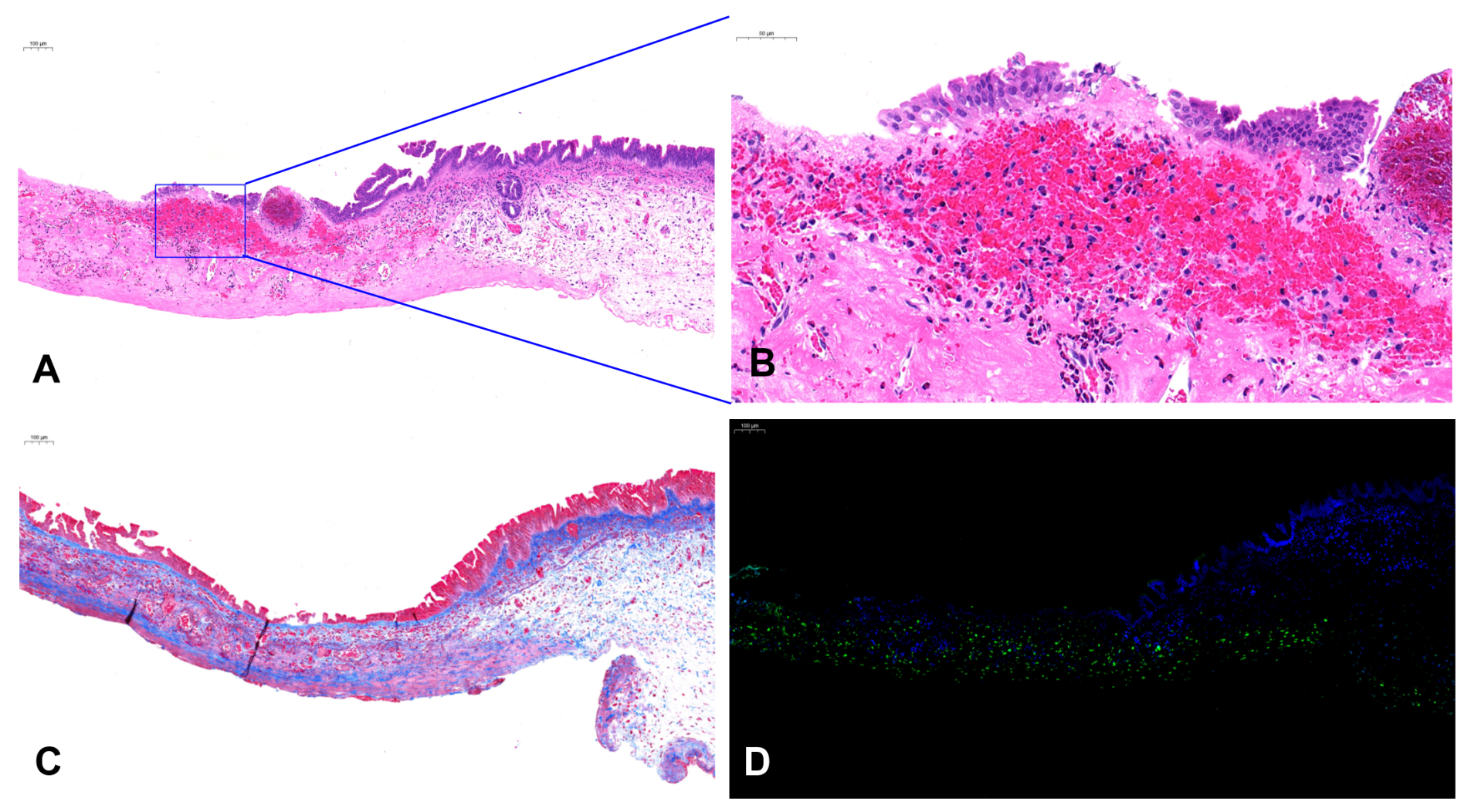

Figure 4

Histopathology analysis from samples 1 day post-IRE. A. The overview of IRE-treated bile duct wall in H\&E staining. The boundary between treatment area and surrounding normal tissue was sharp and clear (arrow: ablation area; line: the boundary of ablation area; magnification: 100x). B. Magnified image of mucosal layer (magnification: 200x). C. The overview of IRE-treated bile duct wall in MT staining. ECM in ablated region was intact and preserved (magnification: 100x). D. Diffuse positive cells in ablated area were observed with demarcated boundary (bright green dots indicate apoptotic cells; magnification: $100 \times)$.
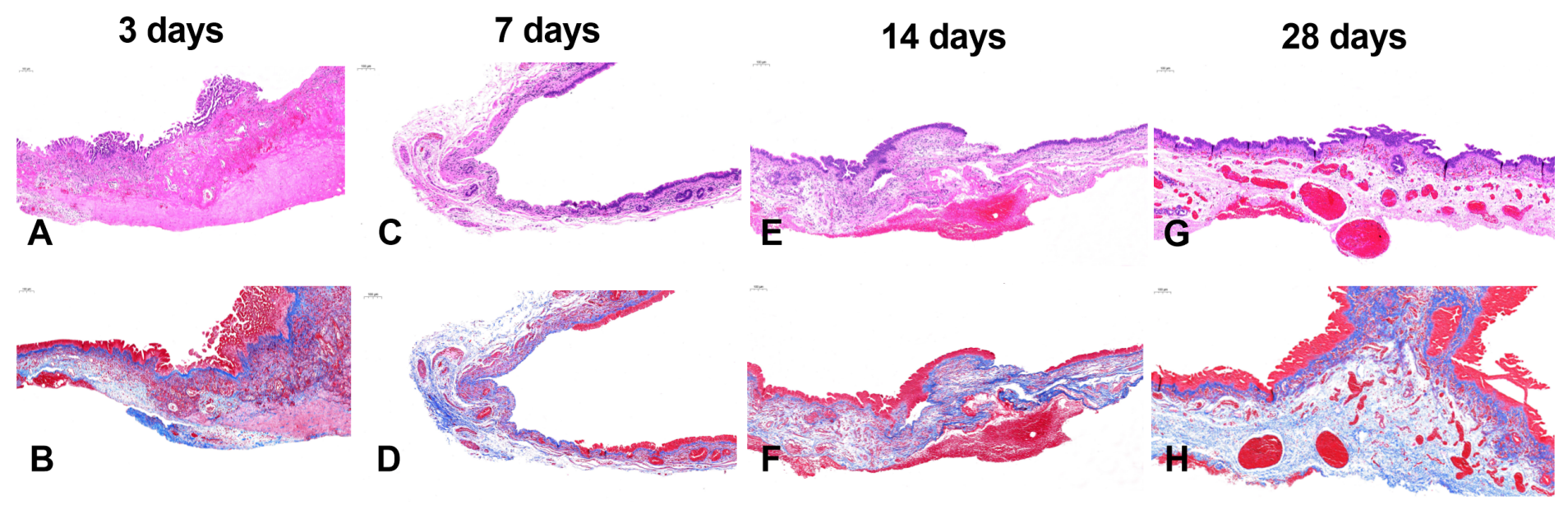

Figure 5 
Histopathology analysis from samples more than 3 days post-IRE. A. H\&E staining on day 3 post-IRE, the full-thickness bile duct ablation was achieved. B. MT staining on day 3 post-IRE. C, D. H\&E staining (C) and MT staining (D) on day 7 post-IRE. Diffuse fibrosis was observed in the treatment region with the missing epithelium. E, F. H\&E staining (E) and MT staining (F) on day 14 post-IRE. G, H. H\&E staining (G) and MT staining $(\mathrm{H})$ on day 28 post-IRE. Regeneration of the bile duct epithelium was completed on day 28 post-IRE. (magnification: $100 \times$ ).

\section{Supplementary Files}

This is a list of supplementary files associated with this preprint. Click to download.

- Supplement1.docx

- SupplementaryFigure2.tif

- SupplementaryFigure1.tif

- ARRIVEGuidelinesChecklist.docx 